\title{
Expression of Cytokeratin 5/6 in Epithelial Neoplasms: An Immunohistochemical Study of 509 Cases
}

Peiguo G. Chu, M.D., Ph.D., Lawrence M. Weiss, M.D.

Department of Pathology, City of Hope National Medical Center, Duarte, California

Cytokeratin 5/6 (CK 5/6) immunoreactivity has been observed in the vast majority of cases of malignant mesothelioma but only rarely in pulmonary adenocarcinomas. Thus, CK 5/6 has been used to distinguish malignant mesothelioma from pulmonary adenocarcinoma. However, the utility of CK $5 / 6$ in distinguishing pleural malignant mesothelioma from pleural metastases from nonpulmonary adenocarcinoma, as well as peritoneal malignant mesothelioma from peritoneal metastatic adenocarcinoma, has not yet been adequately addressed because the tissue expression of CK 5/6 in nonpulmonary neoplasms has not been well defined. We have studied the CK 5/6 expression in 509 cases of various epithelial tumors by immunohistochemistry. We found that the vast majority of cases of squamous cell carcinoma, basal cell carcinoma, thymoma, salivary gland tumor, and biphasic malignant mesothelioma were positive for CK 5/6. In addition, CK 5/6 immunoreactivity was detected in 15 of 24 cases $(62 \%)$ of transitional cell carcinoma, in 5 of 10 cases $(50 \%)$ of endometrial adenocarcinoma, in about one third of cases of pancreatic adenocarcinoma (38\%) and breast adenocarcinoma (31\%), and in one quarter of cases of ovarian adenocarcinomas (25\%). Our study confirms the diagnostic utility of CK 5/6 immunohistochemistry in distinguishing biphasic mesothelioma from pulmonary adenocarcinoma but raises caution about its use for the differential diagnosis of pleural or peritoneal malignant mesothelioma versus pleural or peritoneal metastatic nonpulmonary adenocarcinoma, because many types of nonpulmonary adenocarcinomas may be positive for CK $5 / 6$.

KEY WORDS: Cytokeratin 5/6, Immunohistochemistry, Mesothelioma, Nonpulmonary adenocarci-

Copyright (C) 2002 by The United States and Canadian Academy of Pathology, Inc.

VOL. 15, NO. 1, P. 6, 2002 Printed in the U.S.A.

Date of acceptance: September 18, 2001.

Address reprint requests to: Peiguo G. Chu, M.D., Ph.D., Department of

Pathology, City of Hope National Medical Center, 1500 East Duarte Road,

Duarte, CA 91010. noma, Pulmonary adenocarcinoma.

Mod Pathol 2002;15(1):6-10

Malignant mesothelioma (MM) arises from the serous membranes of various body cavities. About three quarters of MM derive from the pleural cavity; one quarter are from the peritoneal cavity; approximately $2 \%$ of MMs arise in the pericardium; and a smaller number originate in the tunica vaginalis testis $(1,2)$. Unlike the case with other adenocarcinomas, the diagnosis of $\mathrm{MM}$ is rarely made based on routine histology alone and must be confirmed by immunohistochemistry because many mimics can present a similar morphologic appearance at the same anatomic sites (e.g., the pleural and peritoneal cavities) as those typically involved by MM. Distinguishing $\mathrm{MM}$ and adenocarcinoma from various organs has been problematic for years because of the lack of completely sensitive and specific mesothelial markers. In recent years, several monoclonal antibodies have been found to be almost exclusively expressed in MM and only rarely in pulmonary adenocarcinomas, or to be almost exclusively expressed in pulmonary adenocarcinomas and only rarely in MM. The former antibodies include cytokeratin 5/6 (CK 5/6) (3), $N$-cadherin (4), calretinin (5), thrombomodulin (6), MoAb 44-3A6 (7), and $\mathrm{CD} 44 \mathrm{H}$ (8); and the latter antibodies include MOC 31 (9), E-cadherin (10), carcinoembryonic antigen (CEA), BER-ER4, B72.3, BG8, and CD15 (11).

In recent years, CK 5/6 has emerged as one of most useful positive markers for confirming a diagnosis of MM when used in the proper way and on the appropriate setting (12). However, approximately $14 \%$ of adenocarcinomas have been reported also to be positive for CK 5/6 $(3,8)$. Thus, it is important to know which types of adenocarcinomas are positive for CK 5/6 when one is considering use of CK 5/6 antibody to distinguish MM from nonpulmonary adenocarcinoma.

The purpose of the current study is to perform comprehensive testing of CK $5 / 6$ to determine which types of adenocarcinoma may be CK 5/6 positive and to determine the frequency of positivity. 


\section{MATERIALS AND METHODS}

\section{Cases}

509 cases of carcinomas from different organs were selected from our surgical pathology files. The diagnoses were reconfirmed. All tissues had been fixed in $10 \%$ neutral formalin and embedded in paraffin. The organ origins of carcinomas are listed in Table 1 . Of 26 cases of breast carcinoma, 20 cases were ductal and six were lobular carcinoma. Of 28 cases of salivary gland tumors, 11 cases were mixed tumor, nine were adenoid cystic carcinoma, four were Warthin's tumor, three were ductal adenocarcinoma, and one was a mucoepidermoid tumor. Of 54 cases of thyroid tumor, 24 cases were follicular adenoma, 16 were medullary carcinoma, five were follicular carcinoma, and nine were papillary carcinoma. Squamous cell carcinomas were derived from head and neck (11 cases), lung (four cases), and esophagus (10 cases). Of 15 cases of small cell carcinoma, nine were from skin (Merkel cell carcinoma), five from lung, and one from parotid gland.

\section{Immunohistochemistry}

Commercially available CK 5/6 antibody was purchased from Chemicon International, Inc (dilution 1:200, Temecula, CA). Serial $5-\mu \mathrm{m}$ sections were cut from each case. The sections were deparaffinized

TABLE 1. CK 5/6 in Epithelial Neoplasms

\begin{tabular}{|c|c|c|c|c|}
\hline Organs & Tumor Types & $\begin{array}{c}\text { Total } \\
\text { Cases } \\
(n)\end{array}$ & $\begin{array}{c}\text { Positive } \\
\text { Cases } \\
(n)\end{array}$ & $\%$ \\
\hline Skin \& mucosa & Squamous cell carcinoma & 25 & 25 & 100 \\
\hline Skin & Basal cell carcinoma & 20 & 20 & 100 \\
\hline Thymus & Thymoma & 8 & 8 & 100 \\
\hline Salivary gland & All tumors & 28 & 26 & 93 \\
\hline \multirow[t]{2}{*}{ Mesothelioma } & Biphasic & 17 & 13 & 76 \\
\hline & Sarcomatoid & 12 & 3 & 25 \\
\hline Bladder & Transitional cell carcinoma & 24 & 15 & 62 \\
\hline Uterus & Endometrioid carcinoma & 10 & 5 & 50 \\
\hline Pancreas & Adenocarcinoma & 13 & 5 & 38 \\
\hline Breast & Adenocarcinoma & 26 & 8 & 31 \\
\hline Ovary & Adenocarcinoma & 24 & 6 & 25 \\
\hline Liver & Cholangiocarcinoma & 14 & 2 & 14 \\
\hline Lung and GI & Carcinoid tumor & 10 & 1 & 10 \\
\hline Multiple $^{a}$ & Undifferentiated carcinoma & 27 & 2 & 7 \\
\hline Lung & Adenocarcinoma & 21 & 1 & 5 \\
\hline Liver & Hepatocellular carcinoma & 28 & 1 & 4 \\
\hline Adrenal & Cortical tumor & 20 & 0 & 0 \\
\hline Colon & Adenocarcinoma & 20 & 0 & 0 \\
\hline Germ cell & Germ cell tumor & 14 & 0 & 0 \\
\hline Kidney & Carcinoma & 19 & 0 & 0 \\
\hline Lung, liver, \& GI & Neuroendocrine carcinoma & 9 & 0 & 0 \\
\hline Lung \& skin & Small cell carcinoma & 15 & 0 & 0 \\
\hline Prostate & Adenocarcinoma & 18 & 0 & 0 \\
\hline Soft tissue & Epithelioid sarcoma & 12 & 0 & 0 \\
\hline Soft tissue & Synovial sarcoma & 6 & 0 & 0 \\
\hline Stomach & Adenocarcinoma & 15 & 0 & 0 \\
\hline Thyroid & All tumors & 54 & 0 & 0 \\
\hline Total & & 509 & 141 & 27 \\
\hline
\end{tabular}

GI, gastrointestinal tract.

${ }^{a}$ Undifferentiated carcinoma are from lung, stomach, breast, thyroid, nasopharynx, ovary, and parotid gland. and rehydrated in graded alcohol. For heat-induced epitope retrieval, the sections were subjected into 100 mм EDTA buffer (pH 8.0) in a steamer (Black \& Decker, Shelton, CT) at $100^{\circ} \mathrm{C}$ for 20 minutes. The sections were then brought to an automated stainer (DAKO Corporation, Carpinteria, CA) according to the vendor's protocol. EnVision Plus and peroxidase detection methods were used.

Cytoplasmic immunoreactivity was assessed. Only those cases showing $>5 \%$ tumor cell positivity were regarded as positive.

\section{RESULTS}

CK $5 / 6$ positivity was cytoplasmic. The results of staining with CK 5/6 are summarized in Table 1.

\section{CK 5/6 in Malignant Mesothelioma}

Thirteen of 17 cases of biphasic MM (76\%) and 3 of 12 cases of sarcomatoid MM (25\%) were positive for CK 5/6. The CK 5/6 immunoreactivity was mainly identified in the epithelioid component in biphasic MM, whereas rare spindle cells in biphasic MM and in sarcomatoid MM were positive.

\section{CK 5/6 in Carcinomas from Stratified Epithelia}

All 25 cases of squamous cell carcinoma and all 20 cases of basal cell carcinoma showed strong cytoplasmic positivity for CK 5/6 (Fig. 1A-B). There was no difference in staining intensity in carcinomas showing different grades of differentiation or of different origin. Fifteen of 24 cases of transitional cell carcinoma were positive for CK $5 / 6$ (63\%). The CK 5/6 staining pattern and intensity varied between well differentiated and poorly differentiated transitional cell carcinoma. In low-grade papillary
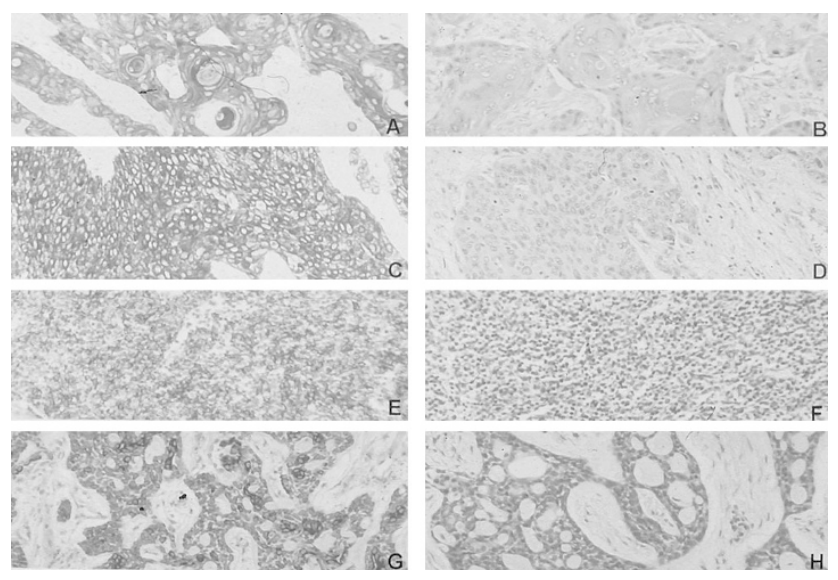

FIGURE 1. CK $5 / 6$ expression in carcinomas from stratified epithelia. The CK 5/6 immunoreactivity is diffuse in squamous cell carcinoma (A), transitional cell carcinoma (C), and thymoma (E), and in adenoid cystic carcinoma of the salivary gland $(\mathbf{G})$. The corresponding hematoxylin and eosin stained sections are shown at the right as $\mathbf{B}, \mathbf{D}$, $\mathbf{F}$, and $\mathbf{H}$, respectively. 
transitional cell carcinoma (TCC), the CK 5/6positive cells were observed at the basal layers of the papillae; whereas in high-grade TCC, tumor cells were diffusely positive for CK 5/6 in some cases (Fig. 1C-D). All eight cases of thymoma were positive for CK $5 / 6$. The positive tumor cells usually formed a network between small lymphocytes (Fig. $1 \mathrm{E}-\mathrm{F})$. Twenty-six of 28 cases of salivary gland tumor were positive for CK 5/6. CK 5/6-positive cells were seen at the basal layers of epithelial projections in Warthin's tumor, spindled (myoepithelial) and glandular cells in mixed tumor, and in all tumor cells in adenoid cystic carcinoma (Fig. 1G-H) and salivary gland ductal adenocarcinoma.

\section{CK 5/6 in Adenocarcinomas from Simple Epithelia}

In contrast to carcinomas of stratified epithelia in which the tumor cells were diffusely positive for CK $5 / 6$, adenocarcinomas were frequently focally positive for CK 5/6. Five of 10 cases of endometrial adenocarcinoma (50\%) and 6 of 24 cases of ovarian adenocarcinoma (25\%) were positive for CK 5/6. Serous papillary (Fig. 2A-B), endometrioid (Fig. 2C$\mathrm{D})$, and clear-cell adenocarcinomas were positive in a significant subset of cases. Eight of 20 cases of invasive breast ductal carcinoma were positive for CK 5/6 (40\%; Fig. 2E-F), whereas none of six cases of lobular carcinoma were positive. Five of 13 cases $(38 \%)$ of pancreatic adenocarcinoma were positive for CK 5/6. In addition, occasional cases of undifferentiated carcinoma $(2 / 27)$, cholangiocarcinoma (2/14), hepatocellular carcinoma (1/28), carcinoid tumor $(1 / 10)$, and pulmonary adenocarcinoma (1/ 21) were focally positive for CK 5/6. In undifferen-
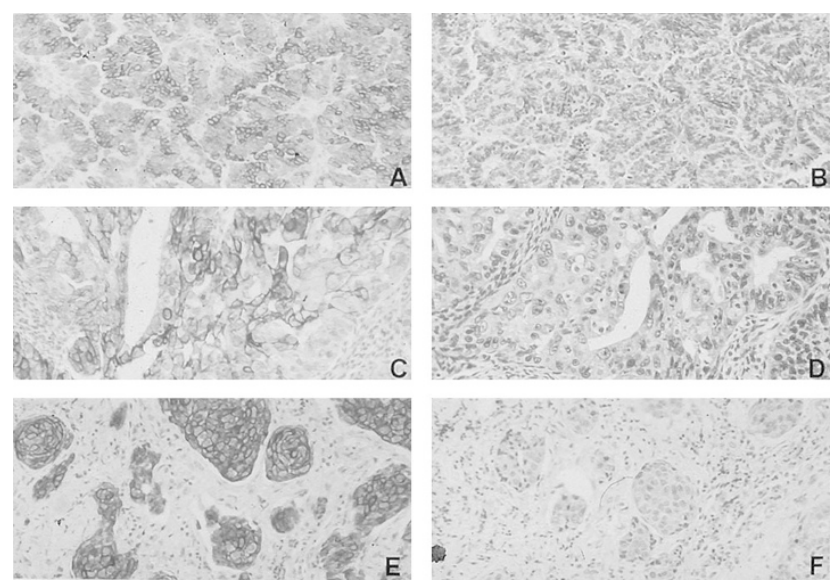

FIGURE 2. CK 5/6 expression in adenocarcinomas from simple epithelia. The CK 5/6 immunoreactivity in a serous papillary adenocarcinoma of the ovary (A) and is focally positive in an endometrioid adenocarcinoma of the uterus (C) and is diffuse in an infiltrating ductal carcinoma of the breast $(\mathbf{E})$. The corresponding hematoxylin and eosin stained sections are shown at the right as B, D, and $\mathbf{F}$, respectively. tiated carcinomas, CK 5/6 positivity was found in areas with squamous differentiation.

In prostate, all 18 cases of adenocarcinoma were negative for CK 5/6, whereas CK 5/6 highlighted basal cells of normal prostatic glands, resembling the staining pattern of high molecular weight keratin as identified by antibody $34 \beta \mathrm{E} 12$ in normal prostate tissue. As in prostate, CK $5 / 6$ also stained myoepithelial cells of breast lobules and ducts, as well as intraductal carcinoma.

There was no CK 5/6 immunoreactivity detected in adrenal cortical tumor $(0 / 20)$, colon adenocarcinoma $(0 / 20)$, gastric adenocarcinoma $(0 / 15)$, thyroid tumors $(0 / 54)$, small cell carcinoma (from skin, lung, and parotid gland) (0/15), large cell neuroendocrine carcinoma $(0 / 9)$, renal cell carcinoma $(0 /$ 19), germ cell tumor $(0 / 14)$, and soft-tissue sarcoma with an epithelioid component (epithelioid sarcoma and biphasic synovial sarcoma; 0/21).

\section{DISCUSSION}

CK5/6 are intermediate-sized basic keratins. In normal tissue, CK5/6 are mainly expressed in keratinizing (epidermis) and nonkeratinizing (mucosa) squamous epithelium, as well as in basal-myoepithelial cell layer of the prostate, breast, and salivary glands. CK5/6 are also seen in benign and malignant tumors of epidermal, squamous mucosal, and myoepithelial origins $(13,14)$. In 1989, Moll et al. (15) demonstrated CK 5 protein in 12 of 13 cases of biphasic malignant mesothelioma (MM) but in none of 21 cases of pulmonary adenocarcinomas. This work did not draw most pathologists' attention until approximately 10 years later, when Ordóñez (3) found that all of 40 cases of mesothelioma and none of 30 cases of pulmonary carcinomas were positive for CK $5 / 6$. Additional study by Cury et al. (8) confirmed these observations. CK 5/6 is currently used by many pathologists as one of several mesothelial markers in the immunohistochemical differential diagnosis of MM versus adenocarcinoma.

Two issues should be considered when using CK $5 / 6$ as an aid to the differential diagnosis of MM versus pleural metastatic tumors. First, the positive rate of CK 5/6 in sarcomatoid MM is much lower (30\%) than that found in biphasic MM (76\%). Although the current study and the study by Attanoos et al. (16) showed that only one third of cases of sarcomatoid mesothelioma expressed CK 5/6, they can usually be distinguished from metastatic adenocarcinoma by routine histologic examination. In fact, sarcomatoid MM is more often confused with primary or metastatic pleural or peritoneal-based sarcomas. In this case, other mesothelial markers, such as thrombomodulin and calretinin, should be 
TABLE 2. Summary of Reported CK 5/6-Positive Tumors from Simple Epithelia

\begin{tabular}{|c|c|c|c|c|c|c|}
\hline Organs & Tumor Types & Current & $\begin{array}{l}\text { Ordóñez } \\
\text { (3) }\end{array}$ & $\begin{array}{l}\text { Baschinsky et al. } \\
\text { (18) }\end{array}$ & $\begin{array}{l}\text { Cury et al. } \\
\text { (8) }\end{array}$ & $\%$ \\
\hline Pancreas & Adenocarcinoma & $5 / 13$ & ND & $5 / 10$ & $0 / 1$ & 42 \\
\hline Uterus & Endometrioid carcinoma & $5 / 10$ & $2 / 10$ & ND & $1 / 1$ & 38 \\
\hline Ovary & Adenocarcinoma & $6 / 24$ & $10 / 30$ & ND & $1 / 6$ & 28 \\
\hline Breast & Adenocarcinoma & $8 / 26$ & $1 / 18$ & ND & $2 / 21$ & 17 \\
\hline Liver & Cholangiocarcinoma & $2 / 14$ & ND & $2 / 10$ & ND & 17 \\
\hline Lung and GI & Carcinoid tumor & $1 / 10$ & ND & ND & ND & 10 \\
\hline Lung etc. & Undifferentiated carcinoma & $2 / 27$ & ND & ND & ND & 7 \\
\hline Colon & Adenocarcinoma & $0 / 20$ & $0 / 10$ & ND & $3 / 10$ & 7 \\
\hline Liver & Hepatocellular carcinoma & $1 / 28$ & ND & $0 / 10$ & ND & 4 \\
\hline Kidney & Carcinoma & $0 / 19$ & $0 / 10$ & $\mathrm{ND}$ & $1 / 4$ & 3 \\
\hline Lung & Adenocarcinoma & $1 / 21$ & $0 / 30$ & ND & $1 / 19$ & 3 \\
\hline Thyroid & All tumors & $0 / 54$ & $1 / 7$ & ND & ND & 2 \\
\hline Adrenal & Cortical tumor & $0 / 20$ & ND & ND & ND & 0 \\
\hline Germ cell & Germ cell tumor & $0 / 14$ & ND & ND & ND & 0 \\
\hline Lung \& GI & Neuroendocrine carcinoma & $0 / 9$ & ND & $0 / 13$ & ND & 0 \\
\hline Lung \& skin & Small cell carcinoma & $0 / 15$ & $\mathrm{ND}$ & ND & ND & 0 \\
\hline Prostate & Adenocarcinoma & $0 / 18$ & $0 / 8$ & ND & ND & 0 \\
\hline Stomach & Adenocarcinoma & $0 / 15$ & ND & ND & ND & 0 \\
\hline
\end{tabular}

$\mathrm{ND}$, not done. GI, gastrointestinal tract.

used in conjunction with CK 5/6. Attanoos et al. (16) have shown that approximately one third of cases of sarcomatoid mesothelioma expressed at least one of those three markers, whereas other spindle cell sarcomas are virtually always negative.

The second consideration in the use of CK $5 / 6$ as an aid to the diagnosis of $\mathrm{MM}$ is that a variety of adenocarcinomas may also be positive for CK 5/6 (Table 2). Ordóñez (3) and Cury et al. (8) both studied CK 5/6 expression in adenocarcinoma; they showed that approximately $15 \%$ of adenocarcinomas of various tissue origins are positive for CK $5 / 6$. The current study obtained a slightly lower CK 5/6 positive rate of $9 \%$ (Table 1 ), probably because of variation in the types of adenocarcinomas studied. Interestingly, all three studies demonstrated that CK 5/6-positive adenocarcinoma was mainly derived from the uterus, ovary, breast, pancreas, and biliary tract (Table 3). Breast adenocarcinoma may be easily confused with pleural MM when it metastasizes to the pleura. Additional immunohistochemical markers may be helpful, such as estrogen receptor (ER), GCDFP-15, CEA, BerEP4, MOC31, CD15, and BG8 for breast cancer and thrombomodulin and calretinin for MM. It may also be problematic when CK 5/6 is used to help differentiate a peritoneal MM from peritoneal metastatic adenocarcinomas, as metastatic pancreatic, endometrial, or ovarian adenocarcinoma have among

TABLE 3. Expression of CK (Cytokeratin) 5/6, CK 7, and CK 14 in Malignant Mesothelioma, Adenocarcinoma, and Squamous Cell Carcinoma

\begin{tabular}{lccc}
\hline \multicolumn{1}{c}{ Tumor Types } & Cytokeratin 5/6 & Cytokeratin 7 & Cytokeratin 14 \\
\hline MM & $+++/-$ & $+++/-$ & - \\
Adenocarcinoma & $-/+$ & ++++ & - \\
SCC & ++++ & - & ++++ \\
\hline
\end{tabular}

MM, malignant mesothelioma; SCC, squamous cell carcinoma. the highest rates of $\mathrm{CK} 5 / 6$ positivity (30-40\%) among adenocarcinomas arising from simple epithelia. These adenocarcinomas frequently exhibit papillary or glandular features that are also frequently seen in MM. In addition, adenocarcinomas from the pancreas, uterus, and ovary frequently metastasize to the peritoneal cavity and may even express other mesothelial markers, such as thrombomodulin, CD44H, and calretinin (8). Therefore, it may be extremely difficult to distinguish a peritoneal mesothelioma from metastatic adenocarcinoma by histologic and immunohistochemical studies alone. Additional clinical history (asbestos exposure), radiologic findings, and other immunomarkers (ER, CEA, MOC-31, BerEp4, and CD15) may be crucial to establish the correct diagnosis.

Assessment of CK 5/6 immunoreactivity may be useful in several other diagnostic situations. Like other high-molecular weight cytokeratins such as CK 14 (17), CK 5/6 protein is expressed mainly in stratified epithelia and cognate neoplasms (Table 1). Therefore, similar to what has been described with CK 14, CK 5/6 may be used as an aid to the identification of squamous differentiation. CK $5 / 6$ may also be useful in the differential diagnosis of hepatocellular carcinoma (rarely CK $5 / 6$ positive) versus metastatic pancreatic carcinoma (CK 5/6 positive in one third to one half of cases), as first described by Baschinsky et al. (18). Third, CK 5/6 may be useful in the immunodiagnosis of bladder versus prostate carcinoma. The current study demonstrated that $63 \%$ of transitional cell carcinoma were positive for CK $5 / 6$, whereas all cases of prostate adenocarcinomas were CK 5/6 negative. In addition, because CK 5/6 stains basal cells of benign prostatic glands but not malignant glands, CK 5/6 antibodies may be useful in the distinction of benign versus malignant prostate proliferation, similar 
to $34 \beta E 12$ antibody. In fact, Abrahams et al. (19) found that CK $5 / 6$ had superior sensitivity (97\%) as compared with $34 \beta E 12$ (33\%) in reliably staining atrophic prostatic glands.

In summary, the current study has confirmed the utility of CK 5/6 in distinguishing mesothelioma from pulmonary adenocarcinoma and from nonpulmonary adenocarcinoma, if used in conjunction with histology, other immunomarkers, clinical history, and radiological findings. CK 5/6 stains carcinomas from stratified epithelia and myoepithelial cells of various tissue origins; thus, it can be used as a marker for squamous cell carcinoma, basal cell carcinoma, transitional cell carcinoma, salivary gland tumors, and thymoma. CK 5/6 may also be useful in separating benign from malignant prostatic glands. Whether CK 5/6 is a superior marker to $34 \beta \mathrm{E} 12$ for the diagnosis of prostate carcinoma requires additional studies.

\section{REFERENCES}

1. Osteen RT. Peritoneal mesothelioma. In: Antman K, Aisner J, editors. Asbestos-related malignancy. Orlando, FL: Grune \& Stratton, 1987. p. 339-55.

2. McDonald JC, McDonald DA. Epidemiology of mesothelioma from estimated incidence. Prev Med 1977;6:426-46.

3. Ordóñez NG. Value of cytokeratin 5/6 immunostaining in distinguishing epithelial mesothelioma of the pleura from lung adenocarcinoma. Am J Surg Pathol 1998;22:1215-21.

4. Chan JKC. Advances in immunohistochemistry: impact on surgical pathology practice. Semin Diagn Pathol 2000;17: $170-7$.

5. Doglioni C, dei Tos AP, Laurino L. Calretinin. A novel immunocytochemical marker for mesothelioma. Am J Surg Pathol 1996;20:1037-46.

6. Ordóñez NG. The value of the antibodies 44-3A6, SM3, HBME-1, and thrombomodulin in differentiating epithelial pleural mesothelioma from lung adenocarcinoma. Am J Surg Pathol 1997;21:1399-408.

7. Spagnolo DV, Whitaker D, Carrello S. The use of monoclonal antibody 44-3A6 in cell blocks in the diagnosis of lung carcinoma, carcinoma metastatic to lung and pleural malignant mesothelioma. Am J Clin Pathol 1991;95:322-9.

8. Cury PM, Butcher DN, Fisher C, Corrin B, Nicholson AG. Value of the mesothelium-associated antibodies thrombomodulin, cytokeratin 5/6, calretinin, and CD44H in distinguishing epithelioid pleural mesothelioma from adenocarcinoma metastatic to the pleura. Mod Pathol 2000;13:107-2.

9. Ordóñez NG. Value of the MOC-31 monoclonal antibody in differentiating epithelial pleural mesothelioma from lung adenocarcinoma. Hum Pathol 1998;29:166-9.

10. Leers MPG, Aarts MMJ, Theunissen PH. E-cadherin and calretinin: a useful combination of immunohistochemical markers for differentiation between mesothelioma and metastatic adenocarcinoma. Histopathology 1998;32:209-16.

11. Riera JR, Astengo-Osuna C, Longmate JA, Battifora H. The immunohistochemical diagnostic panel for epithelial mesothelioma. Am J Surg Pathol 1997;21:1409-19.

12. Moran CA, Wick MR, Suster S. The role of immunohistochemistry in the diagnosis of malignant mesothelioma. Semin Diagn Pathol 2000;17:178-83.

13. Cooper DS, Schermer A, Sun TT. Classification of human epithelia and their neoplasms using monoclonal antibodies to keratins: strategies, applications and limitations. Lab Invest 1985;52:243-56.

14. Miettinen M. Keratin immunohistochemistry: update on applications and pitfalls. Pathol Annu 1993;28:113-43.

15. Moll R, Dhouailly D, Sun TT. Expression of keratin 5 as a distinctive feature of epithelial and biphasic mesotheliomas. An immunohistochemical study using monoclonal antibody AE 14. Virchows Arch 1989;58:129-45.

16. Attenoos RL, Dojcinov SD, Webb R, Gibbs AR. Antimesothelial markers in sarcomatoid mesothelioma and other spindle cell neoplasms. Histopathology 2000;37:224-31.

17. Chu PG, Weiss LM. Cytokeratin 14 expression in epithelial neoplasms: a survey of 435 cases with emphasis on its value in differentiating squamous cell carcinomas from other epithelial neoplasms. Histopathology 2001;39:9-16.

18. Baschinsky DY, Vlasoff D, Beliaeva IV, Frankel WL. Cytokeratin 5/6 immunostaining in hepatobillary and pancreatic neoplasms [abstract]. Mod Pathol 2001;14:193A.

19. Abrahams NA, Ormsby AO, Brainard J. Cytokeratin 5/6 (CK $5 / 6$ ) is an effective substitute for keratin 903 (K 903) in the differentiation of benign (BG) from malignant glands (MG) in prostate needle biopsies [abstract]. Mod Pathol 2001;14: $100 \mathrm{~A}$. 